\title{
Stochastic Skyline Operator
}

\author{
Xuemin Lin, Ying Zhang, Wenjie Zhang, Muhammad Aamir Cheema \\ The University of New South Wales, Australia \\ \{lxue,yingz, zhangw, macheema\}@cse.unsw.edu.au
}

\begin{abstract}
In many applications involving the multiple criteria optimal decision making, users may often want to make a personal trade-off among all optimal solutions. As a key feature, the skyline in a multi-dimensional space provides the minimum set of candidates for such purposes by removing all points not preferred by any (monotonic) utility/scoring functions; that is, the skyline removes all objects not preferred by any user no mater how their preferences vary. Driven by many applications with uncertain data, the probabilistic skyline model is proposed to retrieve uncertain objects based on skyline probabilities. Nevertheless, skyline probabilities cannot capture the preferences of monotonic utility functions. Motivated by this, in this paper we propose a novel skyline operator, namely stochastic skyline. In the light of the expected utility principle, stochastic skyline guarantees to provide the minimum set of candidates for the optimal solutions over all possible monotonic multiplicative utility functions. In contrast to the conventional skyline or the probabilistic skyline computation, we show that the problem of stochastic skyline is NP-complete with respect to the dimensionality. Novel and efficient algorithms are developed to efficiently compute stochastic skyline over multidimensional uncertain data, which run in polynomial time if the dimensionality is fixed. We also show, by theoretical analysis and experiments, that the size of stochastic skyline is quite similar to that of conventional skyline over certain data. Comprehensive experiments demonstrate that our techniques are efficient and scalable regarding both $\mathrm{CPU}$ and $\mathrm{IO}$ costs.
\end{abstract}

\section{INTRODUCTION}

In a $d$-dimensional space $R^{d}$, the skyline is defined over the given preferences of coordinate values on each dimension (i.e., either smaller or larger coordinate values are preferred). Given two points $x$ and $y$ in $R^{d}, x$ dominates $y$ if $x$ is not worse than $y$ on each dimension and is better than $y$ on one dimension according to the given preferences of coordinate values. Given a set $D$ of points (objects) in $R^{d}$, the preferences across different dimensions may conflict to each other regarding $D$; that is, $D$ does not always contain a point that dominates every other point in $D$. Consequently, a scoring function $f$ is often required to rank the points in $D$ to capture the preferences on each dimension to generate the optimal decision (solution). Without loss of generality, in the rest of paper we assume smaller coordinate values are preferred on each dimension and all points are in $R_{+}^{d}$ (i.e. coordinate values are non-negative). This implies that to capture such preferences on each dimension and make the optimal solution with the maximum score, a scoring function $f$ required to rank objects in $D$ should be decreasing.

The skyline of $D$ consists of the points in $D$ which are not dominated by any other points in $D$. It is well known [4], [22] that $x$ dominates $y$ if and only if for any decreasing multivariate function $f, f(x) \geq f(y)$; this is formally stated in page 266 of [21] if a point is regarded as an uncertain object with only one instance that has the occurrence probability 1 . Therefore, in multi-criteria decision applications when users are not content with being given only one optimal solution and want to make a personal trade-off among different optimal solutions (i.e., with maximal scores) over all possible decreasing scoring functions, the skyline provides the minimal set of candidates by removing the points not preferred by any decreasing scoring functions (users). Skyline computation over certain data has been extensively studied (e.g., [4], [17], [25]).

Probabilistic Skyline. Driven by many recent applications involving uncertain data (e.g. environment surveillance, market analysis, WWW and sensor networks), research in uncertain data management has drawn considerable attention from the database community. The skyline analysis over uncertain data has been firstly proposed in [18] where the possible world semantics is adopted to calculate the probabilities, namely skyline probabilities, of uncertain objects not being dominated by other uncertain objects. In [18], efficient techniques are developed to retrieve uncertain objects with skyline probabilities greater than a given threshold, while [2] provides efficient techniques to compute skyline probabilities for all objects.

Motivation. The research towards the problem of multiple criteria optimization over uncertain objects has a long history in economics, finance, and mathematics; see [11], [14], [21] for example. The expected utility principle is the most popular model [11], [14] to select the optimal uncertain object against multiple criteria. In the light of the expected utility principle, an uncertain object $U$ with the maximum expected utility is the optimal solution; that is, select $U$ to maximize $E[f(U)]$ for a given utility function $f$.

Assume that a head coach wants to select the best highjump athlete from all available athletes in her team to attend an international game and decides to evaluate the athletes against their game performances in the recent years, say the last 3 years, where the performance of each athlete in a game is recorded by the final height $h$ and the total number $t$ of failed trails over all attempted heights before successfully passing the final height - $(h, t)$. To conform with the preference of smaller values, assume that $h$ is recorded into 5 bands instead of actual value: band 1 - within a reach of smashing the current world record, band 2 - world leading heights, band 3 - good, band 4 - fair, band 5 - poor. The coach wants to select a player who is very stable (i.e., $t$ is minimized) and jumps high (i.e., $h$ is minimized). Clearly, these two criteria might conflict with each other. Consequently, a utility function $f=f_{1}(h) \times f_{2}(t)$ may be employed by the coach to evaluate the overall performance of a player in a game, where $f_{1}$ and 
$f_{2}$ are nonnegative decreasing functions, mapping $[1,5]$ to $[0,1]$ and $[0, \infty)$ to $[0,1]$, respectively, with $f_{1}(1)=1$ and $f_{2}(0)=1$. The coach selects the athlete with the maximum value of $\left(f_{1}(h) \times f_{2}(t)\right)$. Nevertheless, the game performance of an athlete may fluctuate from game to game due to various reasons. Therefore, it is important to evaluate players against their game performance statistic distribution. For this purpose, an athlete $U$ may be treated as an uncertain object and her performance at each past game may be treated as an instance in a 2-dimensional space with the same probability to occur if no other information is available. The coach could select a player $U$ such that $E(f(U))$ is maximized. Figure 1 gives a small scale example where 3 players are involved.

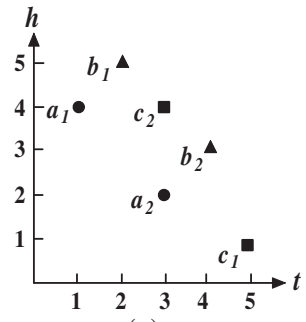

(a)

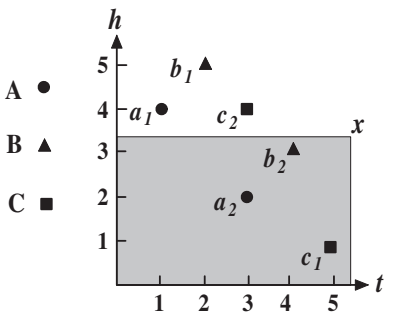

(b)
Fig. 1. Motivating Example

As depicted in Figure 1(a), suppose that $A, B$, and $C$ have 2 instances, respectively. Each instance in objects $A$ and $B$ has the occurrence probability $\frac{1}{2}$. The occurrence probabilities of $c_{1}$ and $c_{2}$ are $\frac{1}{100}$ and $\frac{99}{100}$, respectively, assuming that the player $C$ has the same performance $c_{2}$ for 99 games out of 100 . While choosing an object $U$ from $A, B$, and $C$ to maximize $E[f(U)]$ in the light of the expected utility principle, $E[f(A)]=\frac{1}{2} f_{1}(4) f_{2}(1)+\frac{1}{2} f_{1}(2) f_{2}(3), E[f(B)]=$ $\frac{1}{2} f_{1}(5) f_{2}(2)+\frac{1}{2} f_{1}(3) f_{2}(4)$, and $E[f(C)]=\frac{1}{100} f_{1}(1) f_{2}(5)+$ $\frac{99}{100} f_{1}(4) f_{2}(3)$. Note that nonnegative decreasing utility functions $f_{1}$ and $f_{2}$ could be in any form depending on what kind of risks and trade-offs the coach wants to take; for instance, $f_{1}$ and $f_{2}$ could be in exponential forms such as $f_{1}(h)=e^{a(1-h)}$ and $f_{2}(t)=e^{-b t}$ where $a>0$ and $b>0$ may personally weigh the importance of $h$ and $t$.

Nevertheless, $A$ is always preferred to $B$ since $E[f(A)] \geq$ $E[f(B)]$ for any nonnegative decreasing functions $f_{1}$ and $f_{2}$, respectively. If the coach is taking more risks and only wants to select the athlete with a chance to smash the world record, $f_{1}$ can be defined such that $f_{1}(1)=1$ and $f_{1}(h)=0$ if $h>1$ and $f_{2}$ still uses $e^{-b t}$. If these utility functions are used, then $C$ is the optimal solution since $E[f(A)]$ and $E[f(B)]$ are 0 . This example shows that $B$ is never preferred by any users (i.e. by any such multiplicative decreasing utility functions) and should be excluded as a candidate to any optimal solutions, while $A$ and $C$ should be kept as candidates.

While very useful in applications to determine probabilistic dominance relationships, skyline probabilities cannot capture the preferences of utility (scoring) functions regarding the expected utility principle. Regarding the example in Figure 1(a), it can be immediately verified that the skyline probability of $A$ is 1 , the skyline probability of $B$ is $\frac{1}{2}$, and the skyline probability of $C$ is $\frac{1}{100}$. Clearly, if we choose players based on skyline probability value, then $B$ is always preferred to $C$; that is, there is no chance to exclude the object $B$ without excluding the object $C$. This is an inherent limitation of the probabilistic skyline model.

Stochastic Skyline. Stochastic orders have been widely used in many real-life applications [11], [14], [21], including economics, finance, and multi-criteria statistic decision making. Generally, given a class $\mathcal{F}$ of utility (scoring) functions from all users, an uncertain object (random variable) $U$ stochastically dominates $V$ regarding $\mathcal{F}$, denoted by $U \prec \mathcal{F} V$ if and only if $E[f(U)] \geq E[f(V)]$ for each $f \in \mathcal{F}$ (see [11]); that is, all users prefer $U$ to $V$. Given a set $\mathcal{U}$ of uncertain objects, the stochastic order based skyline, namely stochastic skyline regarding $\mathcal{F}$, is the subset of $\mathcal{U}$ such that each object $U$ in the stochastic skyline is not stochastically dominated by any other object in $\mathcal{U}$ regarding $\mathcal{F}$. Consequently, in applications of multiple criteria optimal decision making over uncertain objects, the stochastic skyline regarding $\mathcal{F}$ provides the minimum set of candidates for the optimal solutions (maximum values), respectively, for all functions in $\mathcal{F}$ by removing the objects not preferred by any function in $\mathcal{F}$.

Several stochastic orders have been defined and their mathematic properties have been well studied in the statistic literature [11], [21]. Consider that multiplicative nonnegative decreasing functions are a very popular family $\mathcal{F}$ of scoring functions to rank an object in $R_{+}^{d}$, that is, $\mathcal{F}=\left\{\prod_{i=1}^{d} f_{i}\left(x_{i}\right)\right\}$ where each $f_{i}$ (for $1 \leq i \leq d$ ) is nonnegative decreasing. In this paper, we investigate the problem of efficiently computing the stochastic skyline regarding lower orthant order [21] since the lower orthant order $\prec_{\mathcal{F}}$ is defined over the family $\mathcal{F}$ of multiplicative decreasing functions. Intuitively, an uncertain object $U$ stochastically dominates $V$ regarding the lower orthant order if and only if for any point $x$ in $R_{+}^{d}$, the probability mass of $U$ is not smaller than the probability mass of $V$ in the region "dominated" by $x$ (i.e. the shaded region in Figure 1(b)); this will be formally defined in Section II. Note that as expected, such stochastic skyline excludes object $B$ and includes $A$ and $C$ in the example in Figure 1(a).

Challenges and Contributions. A main challenge for computing stochastic skyline is how to efficiently check the stochastic dominance relationship between two uncertain objects $U$ and $V$. A naive way to check every point in the whole multi-dimension data space involves an infinite number of points; thus it is computationally infeasible. Efficiently computing stochastic skyline among a large number of objects is even harder. In the paper, following the filtering-verification paradigm we develop novel spatial- and statistics-based filtering techniques to efficiently and effectively eliminate nonpromising uncertain objects. Our filtering techniques are based on an $R$-Tree. We also develop an efficient verification algorithm that only needs to check a finite number of points to verify whether $U$ stochastically dominates $V$.

To the best of our knowledge, this is the first attempt to introduce the stochastic skyline model over uncertain data. Consider that in many applications, probability density func- 
tions (PDFs) of an uncertain object are often described by a set of instances and their occurrence probabilities. In this paper, our investigation focuses on discrete cases of PDFs. Our principal contributions can be summarized as follows.

- We introduce a novel stochastic skyline model on uncertain data with the aim to provide a minimum set of candidates to the optimal solutions over the family of multiplicative decreasing scoring functions for users to make their personal trade-offs.

- We show that the problem of determining the stochastic dominance (i.e. lower orthant order) between two objects is NP-complete regarding the dimensionality, in contrast to the conventional skyline and probabilistic skyline.

- We develop a novel, efficient algorithm to verify if an uncertain object is stochastically dominated by another object. The algorithm runs in polynomial time if the dimensionality is fixed.

- We propose effective and efficient filtering techniques to reduce the number of verifications in computing stochastic skyline.

- We also show, by theoretical analysis and experiments, that the size of stochastic skyline is quite similar to that of skyline over certain data.

Besides the theoretical results, our extensive experiments on real and synthetic data are conducted to demonstrate the efficiency of our techniques.

The rest of the paper is organized as follows. In Section II, we formally define the problem and present preliminaries. In Section III, we show that the problem of determining the stochastic order between two objects is NP-complete regarding the dimensionality $d$ and then present an efficient verification algorithm that runs in polynomial time if $d$ is fixed. Then we present our framework, novel filtering techniques, and a size estimation of stochastic skyline in Section IV, as well as discussions of other stochastic orders and continuous cases of PDFs. Experiment results are presented in Section V. Section VI provides the related work and Section VII concludes the paper.

\section{BACKGROUND INFORMATION}

Table I below summarizes the mathematical notations used throughout this paper.

\begin{tabular}{|c|l|}
\hline Notation & Definition \\
\hline$U U, V$ & uncertain objects in $R_{+}^{d}$ \\
\hline$u, v(x, y)$ & instances (points) of uncertain objects in $R_{+}^{d}$ \\
\hline$n$ & number of uncertain objects \\
\hline$U_{m b b}$ & the MBB of $U$ \\
\hline$U_{\max }\left(U_{\min }\right)$ & upper (lower) corner of the $U_{m b b}$ \\
\hline$R(x, y)$ & rectangular region with $x(y)$ as lower (upper) corner \\
\hline$U . c d f$ & the cumulative distribution function of $U$ \\
\hline$\mu(U)$ & the mean of $U$ \\
\hline$\sigma_{i}^{2}(U)$ & the variance of $U$ on the $i$-th dimension \\
\hline$\prec_{s d} V$ & $U$ stochastically dominates $V$ \\
\hline
\end{tabular}

TABLE I

THE SUMMARY OF NOTATIONS.

\section{A. Problem Definition}

We use $R_{+}^{d}$ to denote the points in $R^{d}$ with nonnegative coordinate values. Without loss of generality, in the paper we only consider the $R_{+}^{d}$ space. A point (instance) $x$ referred in the paper, by default, is in $R_{+}^{d}$.

Let the $i$ th coordinate value of $x$ be denoted by $x_{i}$. Given two points $x$ and $y, x$ dominates $y$ (denoted by $x \prec y$ ) if $x_{i} \leq y_{i}$ for $1 \leq i \leq d$ and there is a $j \in[1, d]$ such that $x_{j}<y_{j}$. We use $x \preceq y$ to denote the case that either $x$ equals $y$ on each dimension or $x$ dominates $y$, and use $R(x, y)$ to denote a rectangular region in $R_{+}^{d}$ where $x$ and $y$ are lower and upper corners, respectively.

An uncertain object can be described either continuously or discretely. As discussed earlier, in this paper we focus on discrete cases and objects are on $R_{+}^{d}$. That is, an uncertain object $U$ consists of a set $\left\{u_{1}, \ldots, u_{m}\right\}$ of instances (points) in $R_{+}^{d}$ where for $1 \leq i \leq m, u_{i}$ is in $R_{+}^{d}$ and occurs with the probability $p_{u_{i}}\left(p_{u_{i}}>0\right)$, and $\sum_{i=1}^{m} p_{u_{i}}=1$. We assume that $m \geq 2$ since $m=1$ means a certain object.

For a point $x \in R_{+}^{d}$, the probability mass $U . c d f(x)$ of $U$ is the sum of the probabilities of the instances in $R((0, \ldots, 0), x)$ where $(0,0, \ldots, 0)$ is the origin in $R^{d}$; that is, $\operatorname{U.cdf}(x)=$ $\sum_{u \preceq x, u \in U} p_{u}$.

Example 1: Suppose the instances of each object in Figure 1(b) have the appearance probabilities as described in the example depicted in Figure 1(a). Then, $A . c d f(x)=\frac{1}{2}$, $B . c d f(x)=\frac{1}{2}$ and $C . c d f(x)=\frac{1}{100}$.

Generally, given an $S \subseteq R_{+}^{d}$, the probability mass of $U$ restricted to $S$ is $\sum_{u \in S \cap U} p_{u}$, denoted by $U . c d f(S)$. The minimal bounding box $U_{m b b}$ of $U$ is the minimal rectangular region $r$ such that $U . c d f(r)=1$. The lower (upper) corner of $U_{m b b}$ is denoted by $U_{\min }\left(U_{\max }\right)$. For presentation simplicity, uncertain objects are sometimes abbreviated to "objects".

Stochastic Order. As discussed in Section I, in this paper we will focus on lower orthant orders [21], called "stochastic dominance" in this paper.

Definition 1 (Stochastic Dominance): Given two uncertain objects $U$ and $V, U$ stochastically dominates an object $V$, denoted by $U \prec_{s d} V$, if $U$.cdf $(x) \geq V$.cdf $(x)$ for any point $x \in R_{+}^{d}$ and $\exists y \in R_{+}^{d}$ such that $U . c d f(y)>\operatorname{V.cdf}(y)$.

Base on the definition, we have that $A \prec_{s d} B, B \nprec_{s d} C$, $C \npreceq_{s d} A$, and $A \npreceq_{s d} C$ regarding the example in Figure 1.

Definition 2 (Stochastic Skyline): Given a set of uncertain objects $\mathcal{U}$, an object $U \in \mathcal{U}$ is a stochastic skyline object if there is no object $V \in \mathcal{U}$ such that $V \prec_{s d} U$. The set of stochastic skyline objects is called the stochastic skyline of $\mathcal{U}$.

Problem Statement. In this paper we investigate the problem of efficiently computing stochastic skyline of a set of uncertain objects.

\section{B. Preliminary}

We say that $U$ stochastically equals $V$, denoted by $U={ }_{s d}$ $V$, if $U . c d f(x)=V \cdot c d f(x)$ for any point $x \in R_{+}^{d}$. Given 
two objects $U$ and $V$, we define $U=V$ if there is a one to one mapping $g$ from $U$ to $V$ such that for each instance $u$ of $U, u=g(u)$ and $p_{u}=p_{g(u)}$; that is, $g$ preserves the locations and probabilities. The following Lemma states that $={ }_{s d}$ is equivalent to $=$ between two uncertain objects; it can be immediately verified based on the definition of $U . c d f$ and V.cdf.

Lemma 1: Given two (uncertain) objects $U$ and $V, U={ }_{s d}$ $V$ if and only if $U=V$.

Minimality of stochastic skyline. The following Theorem is proved in [21] (pp 309).

Theorem 1: Let $U=\left(U_{1}, \ldots, U_{d}\right)$ and $V=\left(V_{1}, \ldots, V_{d}\right)$ be two $d$-dimensional independent random vectors to describe objects $U$ and $V$, respectively, where $U_{i}$ and $V_{i}$ are subvariables of $U$ and $V$ on $i$-dimension. Assuming $U \neq V$, then $U \prec_{s d} V$ if and only if $E\left[\prod_{i=1}^{d} f_{i}\left(U_{i}\right)\right] \geq E\left[\prod_{i=1}^{d} f_{i}\left(V_{i}\right)\right]$ for every collection $\left\{f_{i} \mid 1 \leq i \leq d\right\}$ of univariate non-negative decreasing functions where expectations exist.

Theorem 1 implies that in the light of expected utility principle, the stochastic skyline of $\mathcal{U}$ provides the minimum set of candidates to the optimal solutions, respectively, over all multiplicative decreasing functions by removing the objects not preferred by any multiplicative decreasing function. Here, we say $U$ is preferred to $V$ by $f$ if $E[f(U)] \geq E[f(V)]$.

Framework of computing stochastic skyline. Our techniques for computing stochastic skyline of a set $\mathcal{U}$ of objects follow the standard two phases' framework: filtering and verification. In the filtering phase, efficient filtering techniques are developed to effectively prune non-promising objects. In the verification phase, an efficient algorithm is developed to generate the stochastic skyline of $\mathcal{U}$. The key in the verification phase is to determine whether an uncertain object $U$ stochastically dominates another uncertain object $V$.

\section{Stochastic Dominance Testing}

According to Lemma 1, testing if $U={ }_{s d} V$ is equivalent to testing if $U=V$; thus it can be conducted in $O(d m \log m)$ if instances are firstly sorted according to the lexigraphic order where $m$ is the number of instances in $U(V)$. Given two objects $U$ and $V$, in this section we present an efficient algorithm to determine whether $U$ stochastically dominates $V$ if $U \neq V$. Naively following Definition 1 to compute $U$.cdf $(x)$ and $V \cdot c d f(x)$ for every point $x$ in $R_{+}^{d}$ is computationally infeasible since an infinite number of check points is involved.

\section{A. Testing Finite Number of Points Only}

A point $x \in R_{+}^{d}$ is a violation point regarding $U \preceq_{s d} V$ if $U . c d f(x)<V . c d f(x)$. Assuming that $U \neq V$, it immediately follows from Definition 1 and Lemma 1 that $U$ does not stochastically dominate $V$ if and only if there is a violation point regarding $U \prec_{s d} V$. Consequently, determining if $U$ stochastically dominates $V$ is converted to determining if there is a violation point regarding $U \prec_{s d} V$, given $U \neq V$.

An intuitive way by checking every instance $v$ in $V$ to find a violation point does not work. As depicted in Figure 2,

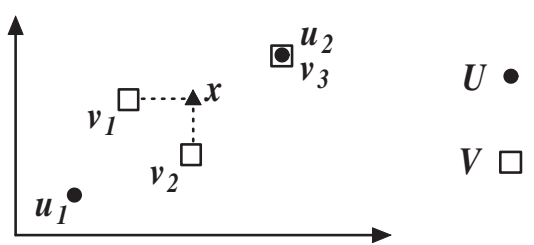

Fig. 2. An Example

$U$ consists of two instances $u_{1}$ and $u_{2}$ with $p_{u_{1}}=p_{u_{2}}=$ $\frac{1}{2}, V$ consists of 3 instances $v_{1}, v_{2}$, and $v_{3}$ with $p_{v_{1}}=$ $p_{v_{2}}=p_{v_{3}}=\frac{1}{3}$, and $v_{3}$ is placed at the same position of $u_{2}$. It can be immediately verified that for $1 \leq i \leq 3$, $U . c d f\left(v_{i}\right) \geq \operatorname{V.cdf}\left(v_{i}\right)$. Nevertheless, we cannot conclude that $U$ stochastically dominates $V$ since $x$ in Figure 2 is a violation point regarding $U \prec_{s d} V$; that is, $V . c d f(x)>U . c d f(x)$.

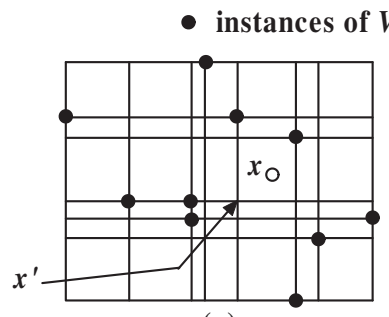

(a)

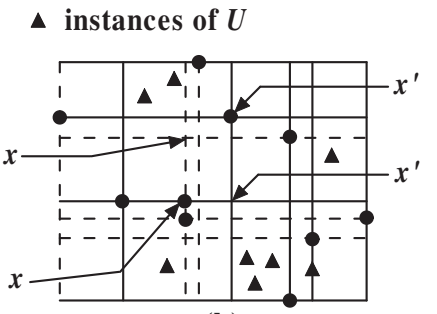

(b)
Fig. 3. Grid Points

For an object $V$ in $R_{+}^{d}$, we use $\mathcal{D}_{i}(V)(1 \leq i \leq d)$ to denote the set of all distinct $i$ th coordinate values of the instances of $V$. Then, $\prod_{i=1}^{d} \mathcal{D}_{i}(V)$ forms a grid; see Figure $3($ a) as an example. Theorem 2 below states that we only need to check every (grid) point in $\prod_{i=1}^{d} \mathcal{D}_{i}(V)$ to determine if there is a violation point regarding $U \prec_{s d} V$.

Theorem 2: Suppose that $U \neq V$. Then, $V$ is not stochastically dominated by $U$ if and only if there is a (grid) point $x \in \prod_{i=1}^{d} \mathcal{D}_{i}(V)$ that is violation point regarding $U \prec_{s d} V$ (i.e., $c d f . V(x)-c d f . U(x)>0$ ).

Proof: The "if" part is immediate according to Definition 1. Below we prove the "only if" part.

Suppose that $V$ is not stochastically dominated by $U$. Then, based on Definition 1 and Lemma 1 there is a violation point regarding $U \prec_{s d} V$. It can be immediately verified that if there is a violation point regarding $U \prec_{s d} V$ then there must be a violation point $x$ in $V_{m b b}$. Let $x^{\prime}$ be the lower corner of the grid cell in $\prod_{i=1}^{d} \mathcal{D}_{i}(V)$ which contains $x$; see Figure 3(a) for example. It is immediate $V . c d f\left(x^{\prime}\right)=V . c d f(x)$ and $U . c d f\left(x^{\prime}\right) \leq U . c d f(x)$. Since $U . c d f(x)<V . c d f(x)$, $U . c d f\left(x^{\prime}\right)<\operatorname{V.cdf}\left(x^{\prime}\right)$.

Naive Algorithm. A naive algorithm is to check every grid point to calculate its U.cdf and V.cdf and terminates when we find a violation point or all grid points are exhausted; it is a correct algorithm, if $U \neq V$, based on Theorem 2. Note that there are at most $m^{d}$ grid points in $\prod_{i=1}^{d} \mathcal{D}_{i}(V)$ where $m$ is the number of instances in $V$. Consequently, the naive algorithm needs to check $O\left(m^{d}\right)$ grid points for computing U.cdf and V.cdf. 


\section{B. NP-completeness}

Below we show that the exponential time complexity regarding $d$ is unavoidable.

Theorem 3: Given two objects $U$ and $V$, assume that $U \neq$ $V$. Then, the problem of determining whether $U \nprec_{s d} V$ is NP-complete regarding the dimensionality $d$.

It is well known [9] that the minimum set cover (decision version) is NP-complete regarding $d$ where $d$ is the number of subsets.

Minimum Set Cover (decision version):

INSTANCE: a collection $C=\left\{S_{i} \mid 1 \leq i \leq d\right\}$ of subsets of $S$ where $S$ contains $(m+1)$ elements, positive integer $K<d$. QUESTION: does $C$ contain a cover for $S$ of size $K$ or less, i.e., a subset $C^{\prime} \subseteq C$ with $\left|C^{\prime}\right| \leq K$ such that every element of $S$ belongs to at least one member (a subset of $S$ ) of $C^{\prime}$ ?

Proof of Theorem 3: We convert the above minimum set cover to a special case of our problem as follows. For each instance of the minimum set cover problem, we construct an instance of our problem as follows. Let $m>1, d>1$, and $S=\left\{u_{i} \mid 0 \leq i \leq m\right\}$.

$U$ has $(m+1)$ instances, $u_{0}, u_{1}, \ldots, u_{m}$ with the occurrence probability $p_{i}(0 \leq i \leq m)$, respectively, where $p_{0}=\frac{d-K}{m d}$, and for $1 \leq i \leq m, p_{i}=\frac{1}{m}-\frac{d-K}{m^{2} d}$. The instances of $U$ are placed in $R_{+}^{d}$ as follows. $u_{0}$ is placed in the origin; that is, $u_{0}=(0,0, \ldots, 0)$. For each $u_{i}(1 \leq i \leq m)$, its coordinate value on each dimension takes either 1 or 2 such that for $1 \leq j \leq d$, the $j$ th coordinate value of $u_{i}$ is 2 if $u_{i} \in S_{j}$ $(1 \leq j \leq d)$, and 1 otherwise.

$V$ has $(d+2)$ instances, $v_{0}, v_{1}, \ldots, v_{d+1}$, with the occurrence probabilities $q_{0}, q_{1}, \ldots, q_{d+1}$, respectively. Here, $q_{0}=\frac{1}{m^{3} d}$, $q_{d+1}=1-\frac{1}{m}-\frac{1}{m^{3} d}$, and for $1 \leq i \leq d, q_{i}=\frac{1}{m d} . v_{0}$ is placed at the origin and $v_{d+1}$ is placed at $(2,2, \ldots, 2)$. For $1 \leq i \leq d$, the $i$ th coordinate value of $v_{i}$ is 2 and the other coordinate values of $v_{i}$ is 1 .

Clearly, $\sum_{i=0}^{m} p_{i}=\sum_{i=0}^{d+1} q_{i}=1, p_{i}>0$ (for $0 \leq i \leq m$ ), and $q_{i}>0$ (for $0 \leq i \leq d+1$ ). Note that for $1 \leq i \leq d$, $\mathcal{D}_{i}(V)=\{0,1,2\}$. For a (grid) point $x=\left(x_{1}, x_{2}, \ldots, x_{d}\right) \in$ $\prod_{i=1}^{d} \mathcal{D}_{i}(V)$, the following can be immediately verified.

1) If $\exists i$ such that $x_{i}=0$, then $c d f . U(x)>c d f . V(x)$; thus it cannot be a violation point regarding $U \prec_{s d} V$.

2) If each $x_{i}=2$ for $1 \leq d$, then $c d f . U(x)=c d f . V(x)=1$; thus it cannot be a violation point regarding $U \prec_{s d} V$.

3) If $x_{i_{j}}=1$ for $1 \leq j \leq l(l>0)$ and $x_{j}=2$ otherwise, then the following two equalities hold where $S^{\prime}=\cup_{j=1}^{l} S_{i_{j}}$.

$$
\begin{gathered}
c d f . V(x)=\frac{d-l}{m d}+\frac{1}{m^{3} d} . \\
c d f . U(x)=\frac{d-K}{m d}+\left|S-S^{\prime}\right|\left(\frac{1}{m}-\frac{d-K}{m^{2} d}\right) .
\end{gathered}
$$

Regarding 3), since $S^{\prime} \subseteq S$, if $S^{\prime} \neq S$ (i.e. $\left\{S_{i_{j}} \mid 1 \leq j \leq\right.$ $l\}$ is not a cover of $S$ ), then $\left|S-S^{\prime}\right| \geq 1$. Consequently, it can be immediately verified that $c d f . U(x)<c d f . V(x)$ if and only if $l \leq K$ and $S^{\prime}=S$; that is, $C^{\prime}=\left\{S_{i_{j}} \mid 1 \leq j \leq l\right\}$ is a cover of $S$ with size $K$ or less. Therefore, the minimum set cover problem has a positive answer if and only if in its corresponding instance, as constructed above, there is a grid point that is a violation point regarding $U \prec_{s d} V$; that is, if and only if $U$ does not stochastically dominates $V$ (by Theorem 2).

\section{Efficient Testing Algorithm}

The NP-completeness implies that the exponential time complexity regarding $d$ cannot be improved. In this section, we present two techniques that may potentially reduce the number of grid points to be tested in practice.

Switching Distinct Values Only. For $1 \leq i \leq d$, let the set of distinct values of $V$ on $i$ th dimension, $\mathcal{D}_{i}(V)=$ $\left\{a_{1}, a_{2}, \ldots, a_{l}\right\}$, be sorted increasingly; that is, $a_{i}<a_{i+1}$ for $1 \leq i \leq l-1$. $a_{j}(1 \leq j \leq l)$ is a switching distinct value regarding $U$ if there is at least one value in $\mathcal{D}_{i}(U)$ that is in $\left[a_{j}, a_{j+1}\right]$ when $j<l$ or in $\left[a_{l}, \infty\right]$ when $j=l$. Let $D_{i}^{s}(V)$ denote the set of switching distinct values of $\mathcal{D}_{i}(V)$ regarding $U$. The following theorem implies that only the (grid) points in $\prod_{i=1}^{d} D_{i}^{s}(V)$, instead of $\prod_{i=1}^{d} \mathcal{D}_{i}(V)$, need to be checked. For example, the grid framed by the solid lines in Figure 3(b) depicts $\prod_{i=1}^{d} D_{i}^{s}(V)$. Clearly, $D_{i}^{s}(V) \subseteq \mathcal{D}_{i}(V)$; consequently, we may reduce significantly the number of grid points for testing.

Theorem 4: Assume that $U \neq V$. Then, $V$ is not stochastically dominated by $U$ if and only if there is a (grid) point $x \in \prod_{i=1}^{d} D_{i}^{s}(V)$ such that $c d f . V(x)-c d f . U(x)>0$.

Proof: $\quad$ Note that $\prod_{i=1}^{d} D_{i}^{s}(V) \subseteq \prod_{i=1}^{d} \mathcal{D}_{i}(V)$. It can be immediately verified that for each grid point $x \in$ $\prod_{i=1}^{d} \mathcal{D}_{i}(V)$ but not in $\prod_{i=1}^{d} D_{i}^{s}(V)$, there is a grid point $x^{\prime}$ in $\prod_{i=1}^{d} D_{i}^{s}(V)$ such that $x \prec x^{\prime}$ and the instances of $U$ falling in $R((0, \ldots, 0), x)$ are the same as those of $U$ falling in $R\left((0, \ldots, 0), x^{\prime}\right)$; see such two pairs in Figure 3(b) for an example. Therefore, $U . c d f\left(x^{\prime}\right)=U . c d f(x)$ and $V . c d f\left(x^{\prime}\right) \geq$ $V . c d f(x)$. Thus, this theorem immediately follows from Theorem 2 .

Discarding a Rectangular Region. Let $R(x, y)$ denote a rectangular region in $R_{+}^{d}$ where the lower and upper corners are $x$ and $y$, respectively. Theorem 5 provides a sufficient condition to exclude any point in $R(x, y)$ to be a violation point regarding $U \prec_{s d} V$. Consequently, $R(x, y)$ can be discarded from the procedure of finding a violation point.

Theorem 5: Given an $R(x, y) \in R_{+}^{d}$, suppose that $U . c d f(x) \geq V . c d f(y)$. Then, for every point $z$ in $R(x, y)$ (i.e., $x \preceq z \preceq y), U . c d f(z) \geq V$.cdf $(z)$.

Proof: $\quad$ Since $x \preceq z \preceq y, U . c d f(z) \geq U . c d f(x)$ and $V . c d f(y) \geq V . c d f(z)$. Thus, the theorem holds.

Such an $R(x, y)$ in Theorem 5 is called valid regarding $U \prec_{s d} V$. To facilitate the observation in Theorem 5, our algorithm is partitioning-based, which iteratively divides rectangular regions into disjoint sub-rectangular regions. A job $Q$ maintains a set of disjoint rectangular regions that cannot be discarded by Theorem 5 (i.e., not yet valid regarding 
$\left.U \prec_{s d} V\right)$. Then, for each rectangular region $R(x, y) \in Q$ the algorithm checks if one of the latest generated corner points of $R(x, y)$ is a violation point regarding $U \prec_{s d} V$. If none of them is a violation point, then the algorithm checks if $R(x, y)$ should be discarded or should be split into two disjoint subrectangular regions to be put into $Q$. Note that the corner points of a $R(x, y)$ are the points $\left(z_{1}, z_{2}, \ldots, z_{d}\right)$ such that for $1 \leq i \leq d, z_{i}$ is $x_{i}$ or $y_{i}$ where $x=\left(x_{1}, \ldots, x_{d}\right)$ and $y=\left(y_{1}, y_{2}, \ldots, y_{d}\right)$. The algorithm terminates if a violation point is found or $Q$ is $\emptyset$. Algorithm 1 below presents our partitioning based testing techniques. The algorithm outputs "true" if $U \prec_{s d} V$ and "false" otherwise.

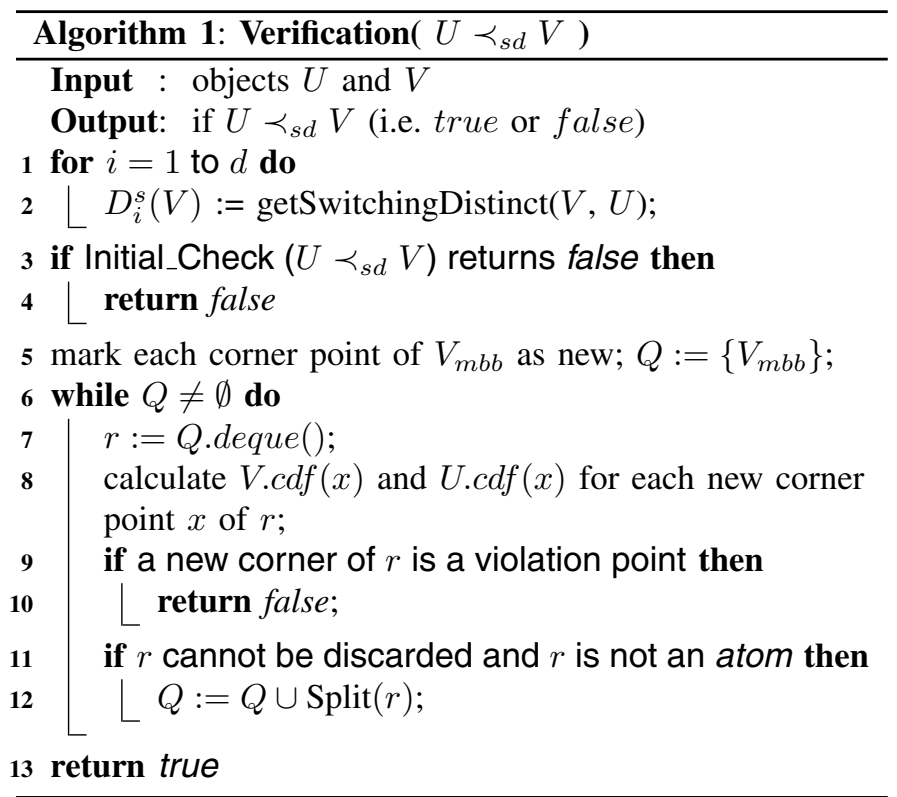

In Algorithm 1, Line 2 is to get the set $D_{i}^{s}(V)$ of switching distinct values of $V$ regarding $U$ on each dimension $i$. Line 3 conducts a quick check on each dimension $i$ as follows. Let $U=\left(U_{1}, \ldots, U_{d}\right)$ and $V=\left(V_{1}, \ldots, V_{d}\right)$ where $U_{i}$ and $V_{i}(1 \leq$ $i \leq d)$ are $i$ th sub-variables of $U$ and $V$, respectively. For each distinct value $a_{j}$ in $D_{i}^{s}(V)(1 \leq i \leq d)$, Initial_Check $\left(U \prec_{s d}\right.$ $V$ ) calculates the total probability, denoted by $\operatorname{V.cdf}\left(V_{i} \leq a_{j}\right)$, of the instances of $V$ with its $i$ th-coordinate value not greater than $a_{j}$; similarly, $U . c d f\left(U_{i} \leq a_{j}\right)$ is also calculated for $U$. If $V . c d f\left(V_{i} \leq a_{j}\right)>U . c d f\left(U_{i} \leq a_{j}\right)$ regarding the currently encountered $a_{j}$, then $U \nprec_{s d} V$ and Initial_Check $\left(U \prec_{s d} V\right)$ returns false. The correctness of Initial_Check $\left(U \prec_{s d} V\right)$ immediately follows from Definition 1.

Line 8 calculates $V . c d f(x)$ and $U . c d f(x)$ for a new corner $x$ of $r$. Line 9 checks whether one of the new corners (grid points) is a violation point and then removes their "new" marks afterwards. In Line 11, the algorithm checks whether $r$ is valid regarding $U \prec_{s d} V$ (i.e. the condition in Theorem 5) and thus can be discarded. $r$ is an atom if it cannot be further split to generate new grid points; that is, on each dimension $i, r$ contains at most 2 values from $D_{i}^{s}(V)$.

$\operatorname{Split}(r)$ in Line 12 splits $r$ into two subregions as follows. Note that for each dimension $i(1 \leq i \leq d)$, the values in $D_{i}^{s}(V)$ contained by $r$ must be consecutive and are denoted by $r \cap D_{i}^{s}(V)$. Firstly, a dimension $i$ is chosen such that $\mid r \cap$ $D_{i}^{s}(V) \mid$ is maximized. Then, the two median values $l_{1}<l_{2}$ in $r \cap D_{i}^{s}(V)$ are chosen to split $r$ into $r_{1}$ and $r_{2}$; that is, the points in $r$ with the $i$ th coordinate value not greater than $l_{1}$ belong to $r_{1}$, and the others in $r$ belong to $r_{2}$. The two median values are used for splitting since we want to "maximize" the number of grid points to be discarded in case if one of $r_{1}$ and $r_{2}$ is valid regarding $U \prec_{s d} V$. Moreover, $\operatorname{Split}(r)$ also marks the newly generated corners of $r_{1}$ and $r_{2}$; that is, the corners of $r_{1}$ and $r_{2}$ are not the corners of $r$. Note that there is an extreme case; while splitting on the $i$ th dimension, if $r$ contains only 3 values in $D_{i}^{s}(V)$, then splitting $r$ into $r_{1}$ and $r_{2}$ on the $i$ th dimension leads to that one of $r_{1}$ and $r_{2}$ degenerates into a rectangular region in a $(d-1)$ space.

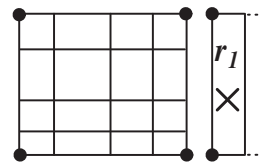

(a)

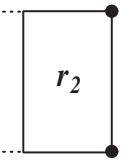

(b)

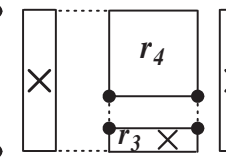

(c) (d)

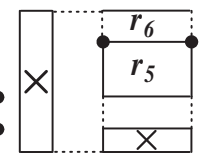

Fig. 4. an example for splitting

Example 2: As depicted in Figure 4(a), assume that $V$ has 5 switching distinct values in each dimension. Algorithm 1 splits $V_{m b b}$ into $r_{1}$ and $r_{2}$ in Figure 4(b). Suppose that $r_{1}$ is discarded by Line 11. Algorithm 1 further splits $r_{2}$ into $r_{3}$ and $r_{4}$ in Figure 4(c). Again, assume that $r_{3}$ is discarded. $r_{4}$ is split into $r_{5}$ and $r_{6}$ by the algorithm where $r_{6}$ is a line segment containing 3 grid points. Any further splitting on $r_{6}$ will be conducted on the line segment. In this example, at each iteration, solid points in Figure 4 indicate the new generated grid points.

Based on the correctness of Initial_Check ( $\left.U \prec_{s d} V\right)$, Theorems 2, 4, and 5, Algorithm 1 is correct when $U \neq V$.

Time Complexity. Algorithm 1 intensively involves the computation of cumulative probabilities of $U$ and $V$ over a rectangular region. To speed up such computation, the instances of each object $U$ are organized by an in-memory aggregate $R$-tree, called local aggregate $R$-tree of $U$, where each entry records the sum of the probabilities of instances contained. Then, the window aggregate techniques in [24] are employed in our algorithm to calculate various $U . c d f$ and $V . c d f$.

getSwitchingDistinct $(V, U)$ is conducted as follows. Note that $\mathcal{D}_{i}(V)$ is sorted increasingly $(1 \leq i \leq d)$. Let $\mathcal{D}_{i}(V)=$ $\left\{a_{1}, \ldots, a_{l}\right\}$. Since the occurrence probability of each instance is positive, based on the definition of switching distinct values it is immediate that $a_{j}(j<l)$ is a switching distinct value if and only if $U . c d f\left(U_{i}<a_{j}\right)<U . c d f\left(U_{i} \leq a_{j+1}\right)$; here, we calculate $U . c d f\left(U_{i} \leq a_{j}\right)$ and $U \cdot c d f\left(U_{i}<a_{j}\right)$ by the window aggregate techniques in [24]. We also calculate $V . c d f\left(V_{i} \leq a_{j}\right)$ for each $a_{j} \in D_{i}^{s}(V)$ to conduct Initial_Check $\left(U \prec_{s d} V\right)$. Clearly, Lines 1-4 totally run in time $O\left(d m \log m+d m\left(T\left(U_{\text {artree }}\right)+T\left(V_{\text {artree }}\right)\right)\right.$ where $T\left(U_{\text {artree }}\right)$ and $T\left(V_{\text {artree }}\right)$ are the costs to conduct window aggregates over the local aggregate $R$-trees of $U$ and $V$, respectively, $m$ is the number of instances in $V$, and $O(m \log m)$ is the time 
complexity to get each $\mathcal{D}_{i}(V)$.

Assuming that $D_{i}^{s}(V)$ is stored in an array. Since it is sorted, it takes constant to find the two splitting values in $\operatorname{Split}(r)$ along a dimension. Since Algorithm 1 calculates U.cdf $(x)$ and $V . c d f(x)$ only once at each grid point $x$ in $\prod_{i=1}^{d} D_{i}^{s}(V)$, the total time spent in calculating $U . c d f$ and $V . c d f$ at all grid points is $O\left(\left(T\left(U_{\text {artree }}\right)+T\left(U_{\text {artree }}\right)\right) \prod_{i=1}^{d} k_{i}\right)$ where $k_{i}=\left|D_{i}^{s}(V)\right|$. Clearly, checking if $r$ is valid regarding $U \prec_{s d} V$ is only invoked when one new grid point (corner) is generated; consequently, the total time for such a check is $O\left(\prod_{i=1}^{d} k_{i}\right)$. Thus, the time complexity from Line 5 to the end is $O\left(\left(T\left(U_{\text {artree }}\right)+T\left(U_{\text {artree }}\right)\right) \prod_{i=1}^{d} k_{i}\right)$. The following theorem is immediate based on the discussions above.

Theorem 6: Algorithm 1 runs in time $O(d m \log m+$ $\left.m^{d}\left(T\left(U_{\text {artree }}\right)+T\left(U_{\text {artree }}\right)\right)\right)$ where $m$ is the number of instances in $V$.

The naive algorithm in Section III-A can also employ the window aggregate techniques in [24] to calculate $U . c d f$ and V.cdf. Although the time complexity of Algorithm 1 is similar to that of the naive algorithm in the worst case, our experiment in Section V demonstrates that the naive algorithm is unpractical, while Algorithm 1 is very efficient in practice.

\section{Stochastic Skyline Computation}

We first present the index to be used, followed by our indexbased framework, filtering techniques, a size estimation of stochastic skyline, and discussions.

\section{A. Statistic R-Tree}

As discussed in Section III-C, the instances of an object are organized into a local aggregate $R$-tree. In our algorithm, we assume that a global $R$-tree is built on the MBBs of each object; that is, the data entries (unit data) in the global $R$ tree are MBBs. To facilitate our filtering techniques, we store the following statistic information at each entry of the global $R$-tree.

Suppose that $U$ has $m$ instances in $R_{+}^{d}, u_{1}, u_{2}, \ldots, u_{m}$ with the occurrence probabilities $p_{1}, p_{2}, \ldots, p_{m}$, respectively.

Definition 3 (mean $\mu$ ): The mean of $U$, denoted by $\mu(U)$, is $\sum_{i=1}^{m} p_{i} \times u_{i}$.

Note that $\mu(U)$ is in $R_{+}^{d}$. For $1 \leq i \leq d, \mu_{i}(U)$ denotes the $i$ th coordinate value of $\mu(U)$.

Definition 4 (variance $\sigma^{2}$ ): For $1 \leq i \leq d, \sigma_{i}^{2}(U)=$ $\sum_{j=1}^{m} p_{j}\left(u_{j, i}-\mu_{i}(U)\right)^{2}$ where each $u_{j, i}$ denotes the $i$ th coordinate value of $u_{j}$.

Suppose that an entry $E$ of the global $R$-tree has $l$ child entries $\left\{E_{1}, E_{2}, \ldots, E_{l}\right\}$. $E$ stores the MBB of each child entry $E_{j}(1 \leq j \leq l)$, as well as $\mu_{i}\left(E_{j}\right)$ and $\sigma_{i}^{2}\left(E_{j}\right)$ for $1 \leq i \leq d$. Here, for $1 \leq i \leq d, \mu_{i}\left(E_{j}\right)=\min \left\{\mu_{i}(V) \mid V \in E_{j}\right\}$ and $\sigma_{i}^{2}\left(E_{j}\right)=\max \left\{\sigma_{i}^{2}(V) \mid V \in E_{j}\right\}$ are called the mean and the variance of $E_{j}$ on $i$ th dimension, respectively.

The global $R$-tree, together with the above statistic information, is called a statistic $R$-tree, denoted by $s R$-tree. Our algorithm for computing stochastic skyline is conducted against $s R$-tree of $\mathcal{U}$. To correctly use the verification algorithm (Algorithm 1), in this paper we assume that no two objects $U$ and $V$ in an $s R$ tree of $\mathcal{U}$ are equal. In case that $\mathcal{U}$ contains equal objects, we only index one of the equal objects and record the object Ids for others while building an $s R$-tree of $\mathcal{U}$.

\section{B. Framework for stochastic skyline computation}

It is immediate that for each point $x \in R_{+}^{d}$, if $U \cdot c d f(x) \leq$ $V . c d f(x)$ and $V . c d f(x) \leq W . c d f(x)$ then $U . c d f(x) \leq$ $W . c d f(x)$. Therefore, $\prec_{s d}$ has the transitivity. Consequently, the standard filtering paradigm is applicable; that is, if $U \prec_{s d}$ $V$ then $V$ can be immediately removed since for any $W$, if $V \prec_{s d} W$ then $U \prec_{s d} W$ and $W$ can be pruned by $U$.

Theorem 7: For two $U$ and $V$, if $\operatorname{dist}\left(U_{\text {min }}\right)<\operatorname{dist}\left(V_{\text {min }}\right)$ then $V \nprec_{s d} U$ where $\operatorname{dist}\left(U_{\text {min }}\right)$ and $\operatorname{dist}\left(V_{\text {min }}\right)$ denote the distances of $U_{\min }$ and $V_{\min }$ to the origin, respectively.

Proof: Immediately, $V_{\text {min }} \nprec U_{\text {min }}$ and $V_{\text {min }} \neq U_{\text {min }}$. Thus, there must be an instance $u$ in $U$ such that $V_{\text {min }} \nprec u$ and $V_{\min } \neq u$. Therefore, $U . c d f(u)>0$ and $\operatorname{V.cdf}(u)=0 . u$ is a violation point regarding $V \prec_{s d} U$.

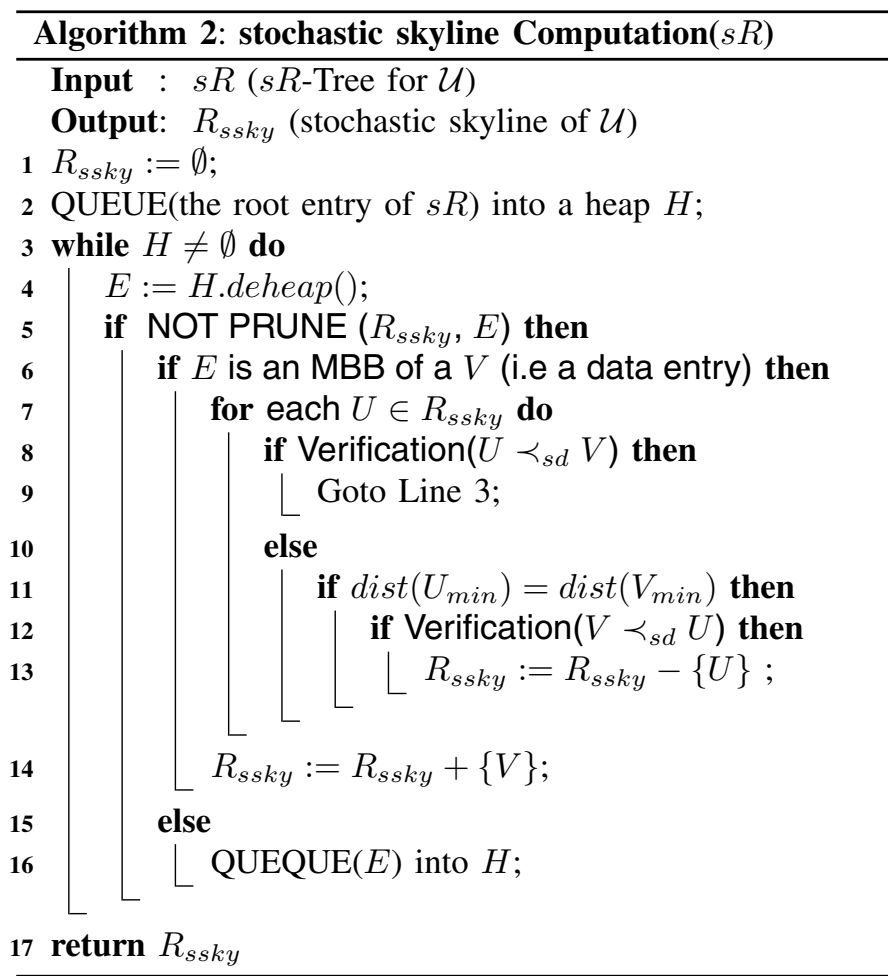

Our index-based algorithm, Algorithm 2 above, adopts the branch and bound search paradigm [17]. It iteratively traverses on the global $s R$-tree to find the data entry (MBB) such that its lower corner has the minimum distance to the origin. An advantage by doing this is that we can guarantee that later accessed objects with distances to the origin is not smaller than those of the early accessed objects. Consequently, based on Theorem 7 below a later accessed object is only possible to stochastically dominate an earlier accessed object when such distances from two objects are the same. Thus, our algorithm has a progressive nature if all such distances are different. 
Lines 2 and 16 push each child entry descriptions of the root or $E$, including its MBBs and the above statistic information, into the heap $H$. Here, $H$ is a min-heap built against the distances of the lower corners of the MBBs of entries to the origin. PRUNE $\left(R_{s s k y}, E\right)$ returns true if $E$ is pruned by the current $R_{\text {ssky }}$ using our filtering techniques in Section IV-C.

Line 8 performs the verification algorithm, Algorithm 1. Since $U$ is accessed earlier than $V, U$ is impossible to be stochastically dominated by $V$ unless $\operatorname{dist}\left(U_{\min }\right)=$ $\operatorname{dist}\left(V_{\text {min }}\right)$ according to Theorem 7 . When $\operatorname{dist}\left(U_{\text {min }}\right)=$ $\operatorname{dist}\left(V_{\min }\right)$, according to our algorithm $U$ is not stochastically dominated by any objects accessed earlier, including those in the current $R_{s s k y}$; nevertheless it is possible that $V$ stochastically dominates $U$ if $V$ is not stochastically dominated by any object in the current $R_{\text {ssky }}$.

\section{Filtering}

A key in Algorithm 2 is to efficiently and effectively conduct $\operatorname{PRUNE}\left(R_{\text {ssky }}, E\right)$. The following two filtering techniques are developed to check if $E$ can be pruned by $U$ for each object $U$ in $R_{s s k y}$ till $R_{s s k y}$ is exhausted or $E$ is pruned.

1. MBB-based Pruning. The following pruning rule is immediate according to the definition of stochastic dominance since each object contains at least 2 instances. Note that $E_{\text {min }}$ denotes the lower corner of the MBB of an entry $E$.

Pruning Rule 1: If $U_{\max } \prec E_{\min }$ or $U_{\max }=E_{\min }$ (i.e. $U_{\max } \preceq E_{\min }$ ), then $U$ stochastically dominates every object in $E$; that is, $E$ can be pruned.

2. Statistic based Pruning. Our statistic based pruning technique uses the observation in Theorem 5 in combining with the Cantelli's Inequality [16].

Suppose that $E$ cannot be pruned by $U$ by Pruning Rule 1; that is, $U_{\max } \nprec E_{\min }$ and $U_{\max } \neq E_{\min }$. Intuitively, $E$ could still be pruned if $U$ and $E$ are "significantly" separated from the statistic point of view; that is, $U_{\max }$ is significantly closer to $E_{\text {min }}$ than the mean of $E$. We show our basic ideas by the case when $E$ is a data entry; that is, $E$ is $V_{m b b}$ - the MBB of an object $V$.

Suppose that $U_{\min } \preceq V_{\min }, U_{\max } \prec V_{\max }$, and $U_{\max } \npreceq$ $V_{\min }$. Let $U_{\max }=\left(a_{1}, a_{2}, \ldots, a_{d}\right), V_{\min }=\left(b_{1}, b_{2}, \ldots, b_{d}\right)$, and $D$ is the subset of $\{1, \ldots, d\}$ such that $a_{i}>b_{i}$ if $i \in D$ and $a_{i} \leq b_{i}$ otherwise. Note that if $D=\emptyset$, then $V$ is pruned by Pruning Rule 1. Figure 5 shows two cases in a 2-dimensional space where $|D|=2$ in Figure 5(a) and $|D|=1$ in Figure 5(b).

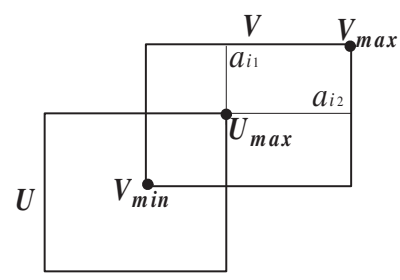

(a)

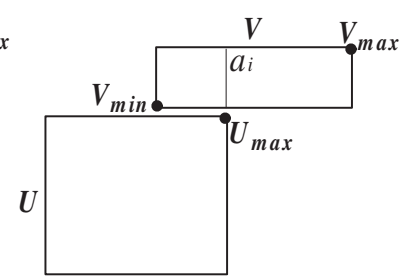

(b)
Fig. 5. Statistic Pruning
Let $k=|D|$. We use the following $k+1$ rectangular regions to cover $V_{m b b}: \forall i \in D, r_{i}=\left\{\left(x_{1}, \ldots, x_{d}\right) \mid x_{i} \leq\right.$ $\left.a_{i},\left(x_{1}, \ldots, x_{d}\right) \in V_{m b b}\right\}$, and the $(k+1)$ th rectangular region is $R\left(U_{\max }, V_{\max }\right)$. Note that for the ease of statistic estimation below, $r_{i}$ and $r_{j}(i, j \in D)$ share a common area. It can be immediately verified that $R\left(U_{\max }, V_{\max }\right)$ is always a valid region regarding $U \prec_{s d} V$. For each $r_{i}(i \in D)$, let the lower-corner and upper-corner of $r_{i}$ be denoted by $r_{i, \min }$ and $r_{i, \max }$, respectively. Clearly, $\forall i \in D, \operatorname{V.cdf}\left(r_{i, \max }\right)=$ $V . c d f\left(V_{i} \leq a_{i}\right)$ where $V_{i}$ is the $i$ th sub-variable of $V$ (i.e. $\left.V=\left(V_{1}, \ldots, V_{d}\right)\right)$, respectively. Therefore, based on Theorems 5 and 2, if $\forall i \in D, r_{i}$ is valid regarding $U \prec_{s d} V$ then $U \prec_{s d} V$ since $\cup_{i \in D} r_{i} \cup R\left(U_{\max }, V_{\max }\right)$ covers $V_{m b b}$. Consider that $V_{\text {min }}=r_{i, \min }(\forall i \in D)$. Immediately, each $r_{i}$ is valid regarding $U \prec_{s d} V$ if and only if

$$
U . c d f\left(V_{\text {min }}\right) \geq \max _{i \in D}\left\{V \cdot c d f\left(V_{i} \leq a_{i}\right)\right\}
$$

In our pruning technique, we can precisely calculate $U . c d f\left(V_{\text {min }}\right)$ for both cases since $U \in R_{s s k y}$ is already read in memory; and the Cantelli's inequality [16] is employed to provide an upper-bound for $V \cdot c d f\left(V_{i} \leq a_{i}\right)$. Let $\delta(x, y)$ be $\frac{1}{1+\frac{x^{2}}{y^{2}}}$ if $y \neq 0,1$ if $x=0$ and $y=0$, and 0 if $x \neq 0$ and $y \stackrel{y^{2}}{=} 0$.

Theorem 8 (Cantelli's Inequality [16]): Suppose that $t$ is a random variable in $R^{1}$ with mean $\mu(t)$ and variance $\sigma^{2}(t)$, $\operatorname{Prob}(t-\mu(t) \geq a) \leq \delta(a, \sigma(t))$ for any $a \geq 0$, where $\operatorname{Prob}(t-\mu(t) \geq a)$ denotes the probability of $t-\mu(t) \geq a$.

Note that Theorem 8 extends the original Cantelli's Inequality [16] to cover the case when $\sigma=0$ and/or $a=0$. The following theorem provides an upper-bound for $\operatorname{Prob}(t \leq b)$ when $b \leq \mu$.

Theorem 9: Assume that $0 \leq b \leq \mu(t)$. Then, $\operatorname{Prob}(t \leq$ $b) \leq \delta(\mu(t)-b, \sigma(t))$.

Proof: Let $t^{\prime}=2 \mu(t)-t$. It can be immediately verified that $\sigma^{2}\left(t^{\prime}\right)=\sigma^{2}(t)$ and $\mu(t)=\mu\left(t^{\prime}\right)$. Applying Cantelli's Inequality on $t^{\prime}$, the theorem holds.

Now we generalize the above observations formally into our second punning rule. Let $U_{\max }=\left(a_{1}, a_{2}, \ldots, a_{d}\right), E_{\min }=$ $\left(b_{1}, b_{2}, \ldots, b_{d}\right)$, and $D$ is the subset of $\{1, \ldots, d\}$ such that $a_{i}>b_{i}$ if $i \in D$ and $a_{i} \leq b_{i}$ otherwise. Let $\Delta(E, U)=$ $\max _{i \in D}\left\{\delta\left(\mu_{i}(E)-a_{i}, \sigma_{i}(E)\right)\right\}$ if $a_{i} \leq \mu_{i}(E)$ for each $i \in D$; and $\Delta(E, U)=\infty$ otherwise.

Pruning Rule 2: Suppose that $U_{\min } \preceq E_{\min }$ and $U_{\max } \prec$ $E_{\text {max }}$. If $U . c d f\left(E_{\text {min }}\right) \geq \Delta(E, U)$, then every object in the entry $E$ of the global $R$-tree is stochastically dominated by $U$; that is $E$ can be pruned by $U$.

Proof: Note that each uncertain object $V$ can also be regarded as a random variable $\left(V_{1}, V_{2}, \ldots, V_{d}\right)$. Immediately, each sub-variable $V_{i}$ of $V$ can be regarded as a random variable in $R^{1}$ with the mean $\mu_{i}(V)$ and the variance $\sigma_{i}^{2}(V)$. From Theorem 5, the inequalities in (3), the assumptions in Section IV-A that no objects indexed by $s R$-tree are equal, and Theorem 9, this Pruning Rule immediately holds if $E$ is 
a data entry $V_{m b b}$; that is, $U \prec_{s d} V$.

Secondly, suppose that $E$ is an intermediate entry of the global $s R$-tree. Since $\Delta(E, U) \neq \infty, a_{i} \leq \mu_{i}(E)$ for $i \in$ $D$. Consider that $\delta(\mu-a, \sigma)$ is increasing and decreasing regarding $\sigma$ and $\mu$, respectively, when $\mu-a \geq 0$ and $\sigma \geq$ 0 , and $\sigma_{i}^{2}(E)$ and $\mu_{i}(E)$ are chosen as the maximum and minimum values among the objects contained. Immediately, $U \prec_{s d} V$ for each $V \in E$ because if $V$ does not satisfy the conditions in Pruning Rule 1 then $U . c d f\left(E_{\text {min }}\right) \geq \Delta(E, U)$ ensures that the inequality (3) holds between $U$ and $V$.

\section{Analysis of Algorithm 2}

$\operatorname{Prune}\left(R_{s s k y}, E\right)$ in Algorithm 2 is conducted as follows that for each object $U$ in $R_{s s k y}$, we first check Pruning Rule 1 and then Pruning Rule 2. It immediately terminates and returns true if $E$ is pruned. Clearly, Pruning Rule 1 runs in time $O(d)$ and Pruning Rule 2 runs in time $O\left(d+T\left(U_{\text {artree }}\right)\right)$ for each $U \in R_{\text {ssky }}$.

Correctness. Based on the correctness of our verification algorithm in Section III-C, and proofs of Pruning Rules 1 and 2 , it can be immediately shown that Algorithm 2 is correct.

Access Order of $R_{s s k y}$. In Algorithm 2, the objects in $R_{\text {ssky }}$ can be accessed in any order. Nevertheless, in our implementation, we access objects $U$ in $R_{s s k y}$ according to the increasing order of $\operatorname{dist}\left(U_{\text {min }}\right)$ with the aim to maximize the chance that Verification $(U, V)$ may terminate earlier and an entry may be pruned earlier.

\section{E. Discussions}

Size Estimation. It tends to be quite complicated to estimate the size of stochastic skyline of $\mathcal{U}$ when each object is described by discrete cases. Below, we show that if each object $U \in \mathcal{U}$ follows a continuous distribution with the uniform assumption, the expected number of stochastic skyline objects is bounded by $\ln ^{d}(n) /(d+1)$ ! - the expected size of conventional skyline in $(d+1)$ dimension space [7], where $n$ is the number of objects in $\mathcal{U}$. The empirical study in Section $\mathrm{V}$ also shows that the size of stochastic skyline objects in $d$-dimension space is almost between those of conventional skyline in $d$-dimensional space and $(d+1)$-dimensional spaces.

Theorem 10: Given a set of objects $\mathcal{U}$, assume that MBBs of all objects are hyper-cubes. We assume the pdfs of an object is continuous with the uniform distribution (i.e., a constant in its $\mathrm{MBB}$ ), and the lengths of the MBBs and lower-corners of the MBBs on each dimension are independent and follow the same distribution. Then the expected size of $\operatorname{ssky}(\mathcal{U})$ is bounded by $\ln ^{d}(n) /(d+1)$ !.

Proof: $\forall U \in \mathcal{U}$, let $l(U)$ denote the length of the hyper-cubes respectively. Clearly, $\left(U_{\min }, l(U)\right)$ is a point in $(d+1)$-dimensional space. Since the pdf of each object follows the uniform distribution, it can be immediately verified that if $\left(U_{\min }, l(U)\right) \prec\left(V_{\min }, l(V)\right)$, then $U$ stochastically dominates $V$. Consequently, the number of objects on stochastic skyline of $\mathcal{U}$ is not greater than the size of skyline of $\left\{\left(U_{\text {min }}, l(U)\right) \mid U \in \mathcal{U}\right\}$. Since $\left\{\left(U_{\text {min }}, l(U)\right) \mid U \in \mathcal{U}\right\}$ is a set of points on a $(d+1)$-dimensional space such that every coordinate is independent and follows the same distribution, the expected skyline size of $\left\{\left(U_{\text {min }}, l(U)\right) \mid U \in \mathcal{U}\right\}$ is bounded by $\ln ^{d}(n) /(d+1)$ ! [7].

Continuous Cases. In the paper, we focus on discrete cases of probability distributions. For continuous cases, we can discrete a continues PDF by sampling methods. While the framework is immediately applicable to the sampled points, the main issue is to estimate the accuracy of a sampling method.

Other Statistic Orders. There are two other popular stochastic orders defined in the literature [21], 1) upper orthant order, and 2) usual stochastic order. We can also define stochastic skyline against these two orders, respectively. Note that the upper orthant order is "symmetric" to the lower orthant order with the preference on larger values; thus the techniques developed in the paper can be immediately modified to the stochastic skyline regarding the upper orthant order.

The usual stochastic order is defined below [21].

Definition 5 (Usual Stochastic Order): Given objects $U$ and $V, U$ dominates $V$, denoted by $U \prec_{s t} V$ if for any upper space $S, U . c d f(S) \leq V . c d f(S)$.

Note that $S \subseteq R^{d}$ is an upper space if for any $x, y \in R^{d}$, $x \preceq y$ and $x \in S$, we have $y \in S$. Similar results to Theorem 1 presented in page 266 of [21] imply that the stochastic skyline regarding the usual stochastic order can provide the minimum sets of candidate for the optimal solutions (with maximum expected values) regarding any forms of decreasing functions; that is, stochastic skyline excludes objects that are not preferred by any decreasing functions.

Note that the number of skyline objects regarding the usual stochastic order is usually larger than the number of skyline objects regarding the lower orthant order, since the lower orthant order may be regarded as a special case of usual stochastic order. However, the size estimation result in Theorem 10 also holds. New techniques for computing the stochastic skyline regarding the usual stochastic order need to be developed. For instance, the verification seems a much more complicated problem.

\section{EMPIRICAL STUDY}

We conduct a thorough performance evaluation on the efficiency and effectiveness of our techniques. Since this is the first work in stochastic skyline computation, our performance evaluation is conducted against our techniques only. We implement the following techniques.

- ssky: Algorithm 2 proposed in Section IV to compute stochastic skyline.

- ssky-NF : ssky without the filtering techniques in Section IV-C but with the non-naive verification algorithm (Algorithm 1) in Section III-C.

- ssky-NV: ssky with the two filtering techniques in Section IV-C and the naive verification algorithm in Section III-A. 


\section{A. Experiment Setup}

All algorithms proposed in the paper are implemented in standard C++ with STL library support and compiled with GNU GCC. Experiments are conducted on a PC with Intel Xeon $2.4 \mathrm{GHz}$ dual $\mathrm{CPU}$ and $4 \mathrm{G}$ memory under Debian Linux. In our implementation, MBBs of the uncertain objects are indexed by an $s R$-tree with page size 2048 bytes. The instances of an object are organized by a main-memory based aggregate $R$-tree with fan-out 8 and we load in the whole aggregate $R$-tree of $V$ if $V$ cannot be pruned by the filtering techniques.

We use both real and synthetic data sets in our evaluation process.

Real dataset is extracted from NBA players' game-by-game statistics (http://www.nba.com), containing 339,721 records of 1,313 players. Each player is treated as an uncertain object where the statistics of a player per game is treated as an instance. For one player, all instances are assumed to take the same probability to appear. In our experiment, we use three attributes, points, assistances, and rebounds in an instance. NBA dataset is employed since the MBBs of players have a very large overlapping degree; thus it may give a good challenge to our techniques.

Synthetic datasets are generated using methodologies in [4] with respect to the following parameters. The centers of objects (objects' MBBs) follow either anti-correlated (anti for short), correlated (corr) or independent (inde) distribution. We use anti as the default distribution for objects' centers. Data domain in each dimension is $[0,1]$. The MBBs of the objects are hype-cube with average edge length $h$ varying from 0.02 to 0.1 with the default value 0.04 . The average number of instances $m$ in each object varies from 200 to 1000 with the default value 400. Locations of instances of an object follow one of the 4 distributions, uniform (unif for short), zipf, constrained normal (norm) or a mixture of the previous three mix. In unif, instances are distributed uniformly inside an MBB with the same occurrence probability. In zipf, firstly an instance $u$ of $U$ is randomly generated and the distances from all other instances to $u$ follow a zipf distribution with $z=0.5$. In norm, instances follow the normal distribution within the MBR of the object with standard deviation $\sigma=0.4 \times h$. In mix, previous three distributions are mixed each with a portion of $\frac{1}{3}$. We use mix as the default distribution for instances. Note that in a synthetic dataset, the instances in every object have the same probability value.

\begin{tabular}{|c|l|}
\hline edge length $h$ & $0.02, \mathbf{0 . 0 4}, 0.06,0.08,0.1$ \\
\hline dimensionality $d$ & $2, \mathbf{3}, 4,5$ \\
\hline number of objects $n$ & $\mathbf{2 0 k}, 40 \mathrm{k}, 60 \mathrm{k}, 80 \mathrm{k}, 100 \mathrm{k}$ \\
\hline number of instances $m$ & $200, \mathbf{4 0 0}, 600,800,1 \mathrm{k}$ \\
\hline object center distribution & anti, corr, inde \\
\hline instance distribution & unif, zipf, norm, mix \\
\hline \multicolumn{2}{|c}{ TABLE II } \\
PARAMETERS
\end{tabular}

We also study the impact of other key parameters. The dimensionality (d) varies from 2 to 5 with the default value 3 .
The number $n$ of objects varies from $20 k$ to $100 k$ where the default value is $\mathbf{2 0 k}$.

Table II summarizes parameter ranges and default values (in bold font). Note that in the default setting, the total number of instances is 8 millions. The maximal number of total instances of the datasets is 40 millions. In the experiments below, these parameters use default values unless otherwise specified.

\begin{tabular}{|c|c|c|}
\hline$p$ & \#psky & \#hit \\
\hline 0.5 & 0 & 0 \\
\hline 0.05 & 83 & 76 \\
\hline 0.005 & 316 & 122 \\
\hline $5 \times 10^{-10}$ & 837 & 124 \\
\hline
\end{tabular}

(a) NBA( \#ssky: 127)

\begin{tabular}{|c|c|c|}
\hline$p$ & \#psky & \#hit \\
\hline 0.8 & 12 & 12 \\
\hline 0.08 & 137 & 94 \\
\hline 0.008 & 310 & 124 \\
\hline $3 \times 10^{-10}$ & 1215 & 147 \\
\hline
\end{tabular}

(b) 3d (\#ssky: 148)
TABLE III

PSKYLINE VS STOCHASTIC SKYLINE

\section{B. Size of Stochastic Skyline}

Table III shows the result sizes (\#psky) of probabilistic skyline and the number (\#hit) of stochastic skyline objects contained by the corresponding probabilistic skyline regarding different probability thresholds. The real dataset NBA data is employed, as well as a 3 dimension synthetic dataset with instance locations following unif and MBB centers following inde (other parameters are default values). The experiment result shows that some stochastic skyline objects may have very small skyline probabilities and some non-stochastic skyline objects may have large skyline probabilities. Consequently, it shows that we may have to use very small probability threshold $(p)$ to generate the probabilistic skyline with large size to cover all stochastic skyline objects.

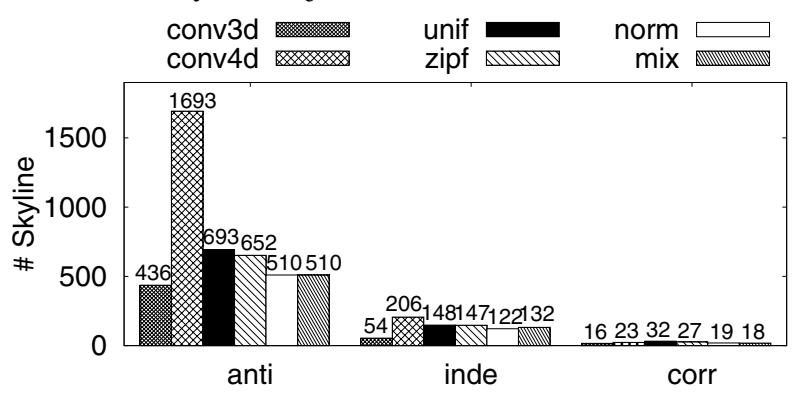

Fig. 6. Skyline Size vs Different Distributions

Figure 6 evaluates the impacts of different distributions of instances locations and object MBB centers, respectively. We use conv to denote the size of (conventional) skyline against the set of MBB centers. The $x$-coordinate in Figure 6 gives different distributions of MBB centers; regarding each distribution of MBB centers, we record the number of skyline objects over one distribution of instance locations. We also record the size of (conventional) skyline against the set of MBB centers in 4-dimensional space to evaluate Theorem 10 in practice since the uncertain data is in 3-dimensional space.

Figure 7 reports the skyline size regarding the number of objects and average number of instances per object. As expected, the skyline size grows with the number of objects but is not very sensitive to the number of instances per object. 


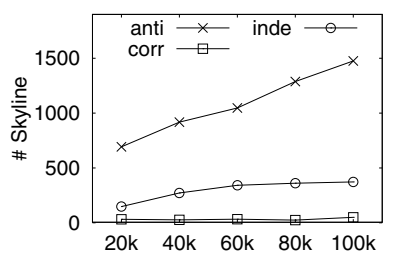

(a) \#Objects

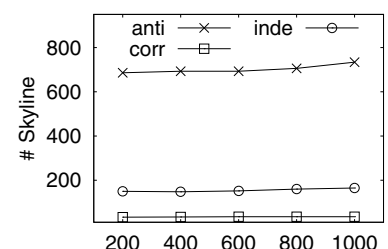

(b) \#Instances
Fig. 7. Skyline Sizes vs Data Sizes

\section{Evaluating Efficiency}

We first evaluate the efficiency of ssky to compute stochastic skyline. We have done the comparison of ssky, ssky-NF and ssky-NV to evaluate the overall performance of filtering techniques and verification techniques. Since the naive verification algorithm is very inefficient comparing with the non-naive verification algorithm Algorithm 1, we use small data size in the experiment where only $10 k$ objects are involved and the average number of instances in Figure 8(b) is 200. As depicted in Figures 8(a)-(b), with naive verification approach, the performance of ssky-NV drops quickly with the increase of instance number and $d$ due to the time complexity $O\left(\mathrm{~m}^{d}\right)$. Some values regarding ssky-NV are missing because under these settings, we can not get result after 5 days running. Moreover, without any filtering techniques, the performance of our algorithm, i.e. ssky-NF algorithm, is very poor due to the large number of IO accesses and verifications. ssky-NF is orders of magnitude slower than ssky.

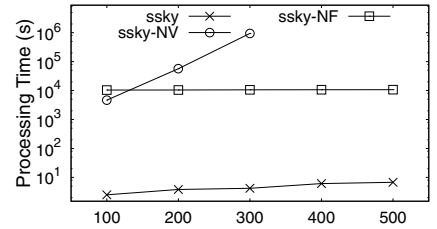

(a) \#Instances

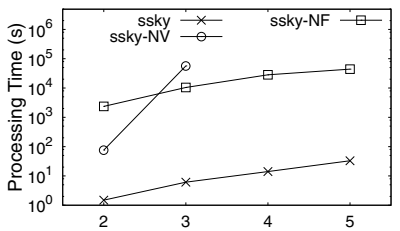

(b) $d$
Fig. 8. Comparison with Naive Techniques

Therefore, in the rest of experiments we no-longer evaluate ssky-NF (without filtering techniques) and ssky-NV (with the naive verification algorithm). We focus on evaluating the two filtering techniques. Particularly, we evaluate the performance of ssky and ssky-F2 where ssky-F2 stands for that in Algorithm 2, only Pruning Rule 1 is used.

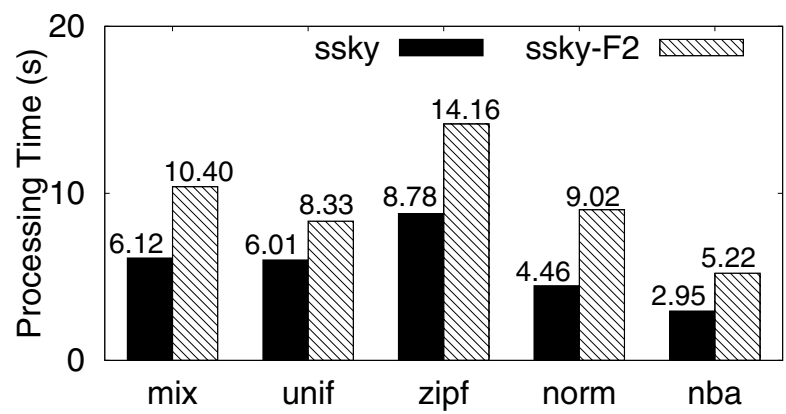

Fig. 9. Processing Time w.r.t Diff. Distributions

Figure 9 reports the evaluation of ssky and ssky-F2 against different distributions of instance locations and NBA data. Both algorithms are quite efficient. It also shows that ssky always significantly outperforms ssky-F2; that is, the Pruning Rule 2 is very effective in practice. Based on the experiment results in Figures 8(a)-(b) which demonstrate that ssky-NF (i.e. without the two filtering techniques) is orders of of magnitude slower than ssky, this experiment implies that the 1st filtering technique is also very effective.

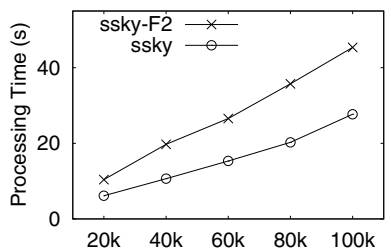

(a) \#Objects

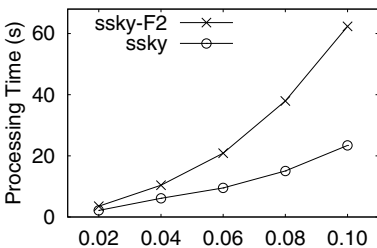

(c) MBR sizes

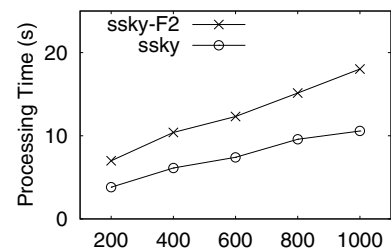

(b) \#Instances

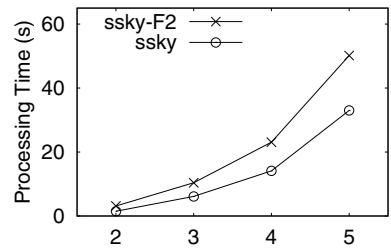

(d) $d$
Fig. 10. Processing Time w.r.t Different Parameters

In Figure 10, we evaluate the scalability of our algorithms against different dataset sizes, number of instances, MBB sizes, and dimensionality. Figure 10(a) and Figure 10(b) show that the performance of ssky and ssky-F2 degrades "linearly" with the growth of dataset size, number of instances, while in Figure 10(c) and Figure 10(d) the performance drops more significantly with the growth of MBB edge size and dimensionality due to the increase of the number of stochastic skyline objects. Nevertheless, the gain of statistics pruning rules becomes more significant when dimensionality is high and MBB edge size is large.

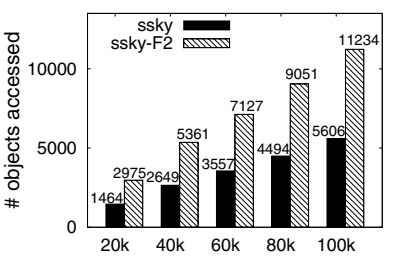

(a) \#Objects

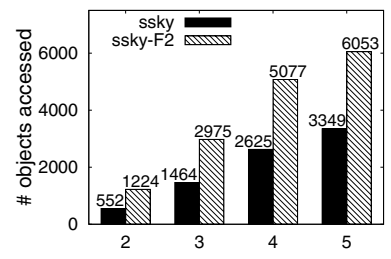

(b) $d$
Fig. 11. I/O costs w.r.t Different Parameters

Figure 11 reports the number of objects accessed regarding dataset sizes and the dimensionality. Both algorithms, ssky and ssky-F2, are I/O efficient because a large number of objects are eliminated by Pruning Rules 1 and/or 2. For instance, Figure 11(a) shows that only $11.2 \%$ and $5.6 \%$ of objects are loaded into main memory by algorithms ssky-F2 and ssky, respectively, when the number of objects is $100 k$. Figure 11 also shows that Pruning Rule 2 can further improve the I/O efficiency. As expected, the number of object (I/O) accesses increases with the increase of dataset size and dimensionality.

Table IV gives a breakdown information of the filtering time and verification (in seconds). The results are reported in where we vary the number of objects from $20 k$ to $100 k$. It shows the filtering costs are much smaller than the costs of verification. 


\begin{tabular}{|c|c|c|c|c|c|}
\hline & $20 \mathrm{k}$ & $40 \mathrm{k}$ & $60 \mathrm{k}$ & $80 \mathrm{k}$ & $100 \mathrm{k}$ \\
\hline Filtering & 0.25 & 0.45 & 0.58 & 0.80 & 2.50 \\
\hline Verification & 5.96 & 10.10 & 17.45 & 20.22 & 31.21 \\
\hline
\end{tabular}

TABLE IV

FILTERING AND VERIFICATION TIME(S)

\section{RELATED WORK}

While the paper is the first work to model and efficiently compute stochastic skyline, below we give a brief overview of skyline computation over conventional and uncertain data, respectively.

Conventional Skyline Computation. Börzsönyi et al [4] firstly study the problem of computing skylines over large datasets. They develop block-nested-loop (BNL) and divideand-conquer (D \& C) based techniques for skyline computation. The Sort Filter Skyline (SFS) algorithm [8] aims to improve BNL by sorting a dataset first. An optimized version of SFS, named linear elimination sort for skyline (LESS) is later proposed in [10]. Sort and limit skyline algorithm (SaLSa) [3] aims to improve SFS and LESS by avoiding scanning the complete set of sorted objects.

The first index based techniques are proposed by Tan et al [23] where two progressive techniques, Bitmap and Index based on bitmap and B-tree structures, are developed. Various index based techniques are also developed (e.g. [12], [13], [17]). Very recently, an effective dynamic indexing technique is proposed in [25] to index the current skyline.

Variations of skyline computation have also been extensively explored; for example, skylines for partially-ordered value domains[6] and skyline cubes [19].

Skyline Computation over Uncertain Data. Considerable research effort has been put into modeling and managing uncertain data in recent years due to many emerging important applications (e.g [1], [5]). Sarma et al [20] purpose to model queries over uncertain data by possible world semantics.

Probabilistic skyline on uncertain data is first tackled by Pei et al [18] where skyline objects are retrieved based on skyline probabilities. Efficient techniques are proposed following the bounding-pruning-refining framework. Lian et al [15] combine reverse skyline with uncertain semantics and study the probabilistic reverse skyline problem in both monochromatic and bichromatic fashion. Atallah and Qi [2] develop sub-quadratic algorithms to compute skyline probabilities for every object. Zhang et al [26] tackle the problem of efficiently on-line computing probabilistic skyline over sliding windows.

\section{CONCLUSION}

In this paper, we propose a novel stochastic skyline model based on the stochastic orders which strictly captures the preference of users and guarantees to provide the minimum set of candidates to the optimal solutions over a broad and popular family of functions. We develop efficient stochastic skyline computation algorithm on large set of objects based on novel filtering and verification techniques. Comprehensive experiments are conducted on both real and synthetic data to demonstrate the efficiency of our techniques. As a possible future work, we will investigate the problem against correlated uncertain data, as well as the stochastic skyline computation over other stochastic orders.

Acknowledgement: This work was partially supported by the three ARC Discovery Grants (DP110102937, DP0987557, DP0881035), and Google Research Award. The first author also acknowledges a general support from NICTA.

\section{REFERENCES}

[1] P. Agrawal, O. Benjelloun, A. D. Sarma, C. Hayworth, S. Nabar, T. Sugihara, and J. Widom. Trio: A system for data, uncertainty, and lineage. In $V L D B, 2006$.

[2] M. J. Atallah and Y. Qi. Computing all skyline probabilities for uncertain data. In PODS, 2009.

[3] I. Bartolini, P. Ciaccia, and M. Patella. Efficient sort-based skyline evaluation. In ACM TODS, 2008.

[4] S. Börzsönyi, D. Kossmann, and K. Stocker. The skyline operator. In ICDE, 2001.

[5] J. Boulos, N. Dalvi, B. Mandhani, S. Mathur, C. Re, and D. Suciu. MYSTIQ: A system for finding more answers by using probabilities. In SIGMOD, 2005.

[6] C.-Y. Chan, P.-K. Eng, and K.-L. Tan. Stratified computation of skylines with paritally ordered domains. In SIGMOD, 2005.

[7] S. Chaudhuri, N. N. Dalvi, and R. Kaushik. Robust cardinality and cost estimation for skyline operator. In ICDE, 2006.

[8] J. Chomicki, P. Godfrey, J. Gryz, and D. Liang. Skyline with presorting. In $I C D E, 2003$.

[9] M. Garey and D. Johnson. Computers and Intractability: A Guider to the Theory of NP-Completeness. 1990.

[10] P. Godfrey, R. Shipley, and J. Gryz. Maximal vector computation in large data sets. In $V L D B, 2005$.

[11] M. Kijima and M. Ohnishi. Stochastic orders and their applications in financial optimization. Mathematical Methods of Operations Research, 50(2), 1999.

[12] D. Kossmann, F. Ramsak, and S. Rost. Shooting stars in the sky: An online algorithm for skyline queries. In $V L D B, 2002$.

[13] K. C. K. Lee, B. Zheng, H. Li, and W. C. Lee. Approaching the skyline in $\mathrm{z}$ order. In $V L D B, 2007$.

[14] H. Levy. Stochastic dominance and exptected utility: survey and analysis. Management Science, 38(4), 1992.

[15] X. Lian and L. Chen. Monochromatic and bichromatic reverse skyline search over uncertain databases. In SIGMOD 2008.

[16] R. Meester. A Natural Introduction to Probability Theory. 2004.

[17] D. Papadias, Y. Tao, G. Fu, and B. Seeger. An optimal progressive algorithm for skyline queries. In SIGMOD, 2003.

[18] J. Pei, B. Jiang, X. Lin, and Y. Yuan. Probabilistic skylines on uncertain data. In $V L D B, 2007$.

[19] J. Pei, Y. Yuan, X. Lin, W. Jin, M. Ester, Q. Liu, W. Wang, Y. Tao, J. X. Yu, and Q. Zhang. Towards multidimensional subspace skyline analysis. ACM Trans. Database Syst., 31(4), 2006.

[20] A. D. Sarma, O. Benjelloun, A. Halevy, and J. Widom. Working models for uncertain data. In ICDE, 2005.

[21] M. Shaked and J. G. Shanthikumar. Stochastic Orders and Their Applications. Academic Press, 2007.

[22] R. E. Steuer. Multi Criteria Optimization: Theory, Computation, and Application. John Willy and Sons, 1995.

[23] K.-L. Tan, P.-K. Eng, and B. C. Ooi. Efficient progressive skyline computation. In $V L D B, 2001$.

[24] Y. Tao and D. Papadias. Range aggregate processing in spatial databases. TKDE, pages 1555-1570, 2004.

[25] S. Zhang, N. Mamoulis, and D. W. Cheung. Scalable skyline computation using object-based space partitioning. In SIGMOD Conference, 2009.

[26] W. Zhang, X. Lin, Y. Zhang, W. Wang, and J. X. Yu. Probabilistic skyline operator over sliding windows. In ICDE, 2009. 